\title{
WEAK SUBORDINATION AND STABLE CLASSES OF MEROMORPHIC FUNCTIONS
}

\author{
BY
}

\author{
KENNETH STEPHENSON
}

\begin{abstract}
This paper introduces the notion of weak subordination: If $F$ and $G$ are meromorphic in the unit disc $U$, then $F$ is weakly subordinate to $G$, written $F \stackrel{w}{\prec} G$, provided there exist analytic functions $\phi$ and $\omega: \mathcal{U} \rightarrow \mathcal{U}$, with $\phi$ an inner function, so that $F \circ \phi=G \circ \omega$. A class $\mathcal{X}$ of meromorphic functions is termed stable if $F \stackrel{w}{\prec} G$ and $G \in \mathcal{X} \Rightarrow F \in \mathcal{X}$.

The motivation is recent work of Burkholder which relates the growth of a function with its range and boundary values. Assume $F$ and $G$ are meromorphic and $G$ has nontangential limits, a.e. Assume further that $F(थ) \cap G(U) \neq \varnothing$ and $G\left(e^{i \theta}\right) \notin F(U)$, a.e. This is denoted by $F<G$. Burkholder proved for several classes $\mathscr{X}$ that

$$
F<G \text { and } G \in \mathcal{X} \Rightarrow F \in \mathcal{X} .
$$

The main result of this paper is the Theorem: $F<G \Rightarrow F \stackrel{w}{\prec} G$. In particular, implication (*) holds for all stable classes $\mathfrak{X}$. The paper goes on to study various stable classes, which include BMOA, $H^{p}, 0<p<\infty, N$, the space of functions of bounded characteristic, and the $M^{\Phi}$ spaces introduced by Burkholder. VMOA and the Bloch functions are examples of classes which are not stable.
\end{abstract}

In this paper we introduce the notion of weak subordination of functions on the unit disc $\mathscr{U}=\{|z|<1\}$ and we investigate classes of functions closed under weak subordination-termed stable classes. Our motivation is recent work of D. L. Burkholder regarding the estimation of growth of functions on the disc from their ranges and boundary behavior. The situations he has considered turn out to be special cases of our more general, function-theoretic notion. We are thus able to extend his results to all stable classes, which include the classes he investigated plus several other standard function spaces.

Let $F$ and $G$ be meromorphic functions in the unit disc and assume that $G\left(e^{i \theta}\right)$ exists as a nontangential limit for almost all $\theta \in[0,2 \pi]$. Assume further that $F(\mathcal{U}) \cap G(\mathcal{U})$ is nonempty and $G\left(e^{i \theta}\right) \notin F(\mathcal{U})$, a.e. For convenience, we indicate this situation by $F<G$. Burkholder strengthened a method of L. Hansen [7] to show that for classes $\mathcal{X}$ of functions satisfying certain growth conditions one can conclude

$$
F<G \text { and } G \in \mathcal{X} \Rightarrow F \in \mathcal{X} .
$$

Burkholder first proved this for $\mathcal{X}=H^{p}, 0<p<\infty$, using Brownian motion

Received by the editors March 13, 1979; presented to the Society November 4, 1978, Charleston, South Carolina.

AMS (MOS) subject classifications (1970). Primary 30A78, 30A20, 30A76.

Key words and phrases. Inner function, composition, subordination, Hardy spaces, BMOA.

(C) 1980 American Mathematical Society 0002-9947/80/0000-0566/\$04.25 
arguments [3]. He subsequently used potential theory and nontangential maximal functions to prove it for more general classes $X=M^{\Phi}$ [4].

We define the notion of weak subordination: If $F$ and $G$ are meromorphic in $\mathscr{U}$, we say $F$ is weakly subordinate to $G$, denoted $F \stackrel{w}{\prec} G$, if there exist analytic functions $\phi, \omega: \mathcal{Q} \rightarrow \mathcal{Q}$, with $\phi$ an inner function, so that $F \circ \phi=G \circ \omega$. A class $\mathcal{X}$ of meromorphic functions is stable if it is closed under weak subordination, that is,

$$
F \stackrel{w}{\prec} G \text { and } G \in \mathcal{X} \Rightarrow F \in \mathcal{X} .
$$

The connection with Burkholder's work is this:

$$
F<G \Rightarrow F \stackrel{\text { w }}{\prec} G \text {. }
$$

In particular, implication (1) holds for all stable classes $\mathfrak{X}$.

The Hardy spaces, $H^{p}, 0<p \leqslant \infty$, the Smirnov class, $N_{*}$, and the space of meromorphic functions of bounded characteristic, $\mathrm{BC}$, were shown to be stable by the author in [17]. We show in $\$ 4$ that BMOA, analytic functions of bounded mean oscillation, is also stable. In $\$ 5$ we prove that the classes $M^{\Phi}$ introduced by Burkholder in [4] are stable if we redefine them slightly to accomodate meromorphic functions; the previous results follow when attention is restricted to holomorphic functions. In $\S 6$ we give examples of spaces which are not stable, and we make some concluding remarks.

The proof of (2) is given in $\$ 2$. The method of proof obscures somewhat the central fact that $F<G$ implies $F(\mathscr{U}) \backslash G(\mathscr{U})$ has logarithmic capacity zero, so an alternate structure theorem is given in $\$ 3$. We then justify our symbols < and $\stackrel{w}{\prec}$ by showing that these relations are transitive. This second approach is also of some independent interest since it allows one to view $F$ and $G$ as maps into the covering surface of $G(\mathcal{U})$, and shows intuitively why $G\left(e^{i \theta}\right) \notin F(\mathcal{U})$, a.e., means $G$ "leaves" the range of $F$ on almost all radii-this was the original insight gained from the Brownian motion approach.

I wish to thank D. L. Burkholder for conversations on this material and specifically for his conjecture that Lemma 6 might hold. It is also interesting to note his suggestion [3, p. 200] of a "kind of subordination of $F$ to $G$ " when $F<G$.

1. Preliminaries. $\mathscr{T}(\mathscr{U})$ and $\mathcal{H}(\mathscr{U})$ denote, respectively, the meromorphic and holomorphic functions on the unit disc. $S^{2}$ is the Riemann sphere, $S^{2}=\mathbf{C} \cup\{\infty\}$. For $f \in \mathfrak{R}(\mathscr{Q}), f\left(e^{i \theta}\right) \in S^{2}$ always denotes the nontangential limit of $f$ at $e^{i \theta}$, indicating in particular that this limit exists. $\mathrm{BC}=\{f \in \mathscr{N}(\mathcal{Q}): f$ is of bounded characteristic \}. If $\Omega \subseteq S^{2}, \partial \Omega$ denotes its boundary. On $\partial \mathscr{Q}$, normalized Lebesgue measure is denoted by $m$, and a.e. means almost everywhere with respect to $m$. $\phi \in \mathcal{H}(\mathcal{Q})$ is an inner function provided $\phi(\mathcal{U}) \subseteq \mathcal{Q}$ and $\left|\phi\left(e^{i \theta}\right)\right|=1$, a.e. A general reference for properties of inner functions is Duren [6].

Definition. Let $F, G \in \Re(\mathcal{U})$. We say $F$ is weakly subordinate to $G$, written $F \stackrel{w}{\prec} G$, if there exists an inner function $\phi$ and an analytic function $\omega$ : $\mathcal{U} \rightarrow \mathcal{Q}$ so 
that $F \circ \phi=G \circ \omega$. This corresponds with the usual notion of subordination when $\phi(z) \equiv z$ and $\omega(0)=0$.

Definition. We say $\mathcal{X} \subseteq \mathscr{T}(\mathscr{Q})$ is stable if it is closed under weak subordination, i.e., if $F \stackrel{w}{\prec} G$ and $G \in \mathcal{X} \Rightarrow F \in \mathcal{X}$. When considering a specific space $\mathfrak{X}$, it is convenient to verify the following two conditions, which can be shown to be both necessary and sufficient for stability:

(a) $F \in \mathcal{X}$ and $\omega: \mathcal{Q} \rightarrow \mathcal{Q} \Rightarrow F \circ \omega \in \mathcal{X}$,

(b) $F \in \mathfrak{N}(\mathscr{Q}), \phi$ an inner function, and $F \circ \phi \in \mathcal{X} \Rightarrow F \in \mathcal{X}$.

For convenience, we now record some lemmas which will be needed at various points in the sequel. A proof of the first can be found in [11]; the next is a generalization of Löwner's theorem [18, Chapter VIII, $\$ \$ 5$ and 6]; the third is proven in [5, Chapter 2, §8].

LemMA 1. Let $\omega: \mathcal{Q} \rightarrow \mathcal{Q}$ be analytic with $\alpha=\omega(0)$. For $f \in H^{2}=H^{2}(\mathcal{U})$,

$$
\|f \circ \omega\|_{2}^{2} \leqslant \frac{1+|\alpha|}{1-|\alpha|}\|f\|_{2}^{2}
$$

Furthermore, if $\omega$ is an inner function, then

$$
\frac{1-|\alpha|}{1+|\alpha|}\|f\|_{2}^{2} \leqslant\|f \circ \omega\|_{2}^{2}
$$

In particular, if $\omega(0)=0$, then $\|f \circ \omega\|_{2} \leqslant\|f\|_{2}$ with equality if $\omega$ is an inner function.

LEMMA 2. Let $\omega: \mathcal{Q} \rightarrow$ Q be analytic. Let $E^{*} \subset \partial \mathscr{Q}$ with $m\left(E^{*}\right)=0$ and define $E=\left\{e^{i \theta}: \omega\left(e^{i \theta}\right) \in E^{*}\right\} \subset \partial \mathscr{Q}$. Then $m(E)=0$.

Lemma 3. Let $A \subset \mathcal{Q}$ be a relatively closed subset of (logarithmic) capacity zero, with $\phi_{A}: \mathscr{U} \rightarrow \mathcal{Q} \backslash A$ a universal covering map. Then $\phi_{A}$ is an inner function.

LEMma 4. Assume $f \in \Re(\mathscr{Q})$ has nontangential limits, a.e., and $\omega: \mathcal{Q} \rightarrow \mathscr{Q}$ is analytic. Let $E \subseteq \partial \mathcal{Q}$ be the set on which $f \circ \omega$ has nontangential limits. Then

$$
(f \circ \omega)\left(e^{i \theta}\right)=f\left(\omega\left(e^{i \theta}\right)\right)
$$

for almost all $e^{i \theta} \in E$.

Proof. Consider the right-hand side of (3). $\omega\left(e^{i \theta}\right)$ exists, a.e., by Fatou's theorem. Since $f(\xi)$ exists for all $\xi \in \mathcal{Q}$ and for almost all $\xi \in \partial \mathscr{Q}$, an application of Lemma 2 implies that $f\left(\omega\left(e^{i \theta}\right)\right)$ makes sense, a.e. The left side of (3) makes sense for all $e^{i \theta} \in E$, so the only way (3) can fail for a point $e^{i \theta}$ is if $f$ has an asymptotic value along the path $\Gamma: r \rightarrow \omega\left(r e^{i \theta}\right)$ which is not the same as the nontangential limit $f\left(\omega\left(e^{i \theta}\right)\right)$. This exceptional set of values $\omega\left(e^{i \theta}\right)$ is countable by Bagemihl's theorem on ambiguous points [5, Theorem 4.12]. Thus, another application of Lemma 2 completes the proof.

\section{Proof of Theorem 1.}

THEOREM 1. $F<G$ implies there exist analytic functions $\phi, \omega: \mathcal{U} \rightarrow \mathcal{Q}$, with $\phi$ an inner function, so that $F \circ \phi=G \circ \omega$. 
Proof. Assume both $F$ and $G$ are nonconstant, for otherwise the result is immediate. $F<G$ implies $F(\mathscr{U})$ intersects $G(\mathscr{U})$ in a nonempty open set. Choose $a, b \in \mathcal{Q}$ with $F(a)=G(b)$ and $G^{\prime}(b) \neq 0$. In a sufficiently small neighborhood $\Omega$, $a \in \Omega \subseteq \mathcal{Q}$, we can define a single-valued analytic function $h=G^{-1}(F)$ with $h(a)=b$.

We can continue $h$ analytically to a maximal connected Riemann surface $W$ lying over $\mathcal{Q}$. Perhaps the easiest way to describe $W$ is to consider $\mathfrak{h}$, the complete analytic function containing the function element $(h, \Omega)$. Let $\mathcal{W}$ be the associated Riemann surface with projection $\pi$ into the complex plane. $W$ is simply a component of $\pi^{-1}(\mathcal{U}) \cap h^{-1}(\mathcal{U}) \subseteq \mathscr{W}$ which contains the branch associated with $(h, \Omega)$. That is, $W$ contains an open set $\tilde{\Omega}$ with $\pi: \tilde{\Omega} \rightarrow \Omega$ a homeomorphism and with $\mathfrak{h}(\xi)=h(\pi(\xi)), \xi \in \tilde{\Omega}$. Let $\tilde{a}$ denote the point of $\tilde{\Omega}$ with $\pi(\tilde{a})=a$.

We continue to use $\pi$ to denote the projection $\pi: W \rightarrow \mathscr{Q}$. Since this is a bounded analytic function on $W$, we see that $W$ has $\mathcal{Q}$ as its universal covering surface. Let $\rho: \mathcal{Q} \rightarrow W$ be a universal covering map with $\rho(0)=\tilde{a}$. Now, define

$$
\phi=\pi \circ \rho \text { and } \omega=\mathfrak{h} \circ \rho .
$$

$\phi$ and $\omega$ are well defined analytic functions from $\mathcal{Q}$ into $\mathcal{Q}$. Let $\xi=\rho(z) \in W$ and consider $G \circ \omega$ on $\rho^{-1}(\tilde{\Omega}) \subseteq \mathscr{U}$ :

$$
\begin{aligned}
(G \circ \omega)(z) & =G[\mathfrak{h}(\rho(z))]=G[\mathfrak{h}(\xi)]=G[h(\pi(\xi))] \\
& =G\left[G^{-1}(F(\pi(\xi)))\right]=F(\pi(\xi))=F(\pi(\rho(z))) \\
& =(F \circ \phi)(z), \quad z \in \rho^{-1}(\tilde{\Omega}) .
\end{aligned}
$$

Therefore, $G \circ \omega \equiv F \circ \phi$ on $\mathcal{U}$. It remains only to show that $\phi$ is an inner function.

Define the following subsets of $\partial \mathscr{Q}$ :

$$
\begin{aligned}
& E_{1}=\left\{e^{i \theta}: \phi\left(e^{i \theta}\right) \text { or } \omega\left(e^{i \theta}\right) \text { fails to exist }\right\}, \\
& E_{2}=\left\{e^{i \theta}:\left|\omega\left(e^{i \theta}\right)\right|<1 \text { and } G^{\prime}\left(\omega\left(e^{i \theta}\right)\right)=0\right\}, \\
& E_{3}=\left\{e^{i \theta} \notin E_{2}:\left|\phi\left(e^{i \theta}\right)\right|<1 \text { and }\left|\omega\left(e^{i \theta}\right)\right|<1\right\}, \\
& E_{4}=\left\{e^{i \theta}:\left|\phi\left(e^{i \theta}\right)\right|<1 \text { and }\left|\omega\left(e^{i \theta}\right)\right|=1\right\} .
\end{aligned}
$$

$\phi$ has unimodular radial limits on $\partial \mathscr{\cup} \cup_{1}^{4} E_{j}$, so it suffices to prove $m\left(E_{j}\right)=0$, $j=1,2,3,4$.

Fatou's theorem implies $m\left(E_{1}\right)=0$ and, by the countability of $\left\{z: G^{\prime}(z)=0\right\}$, $m\left(E_{2}\right)=0$ also. We show, using the maximality of $W$, that $E_{3}=\varnothing$ : Assume $e^{i \theta} \in E_{3}$, with $\alpha=\phi\left(e^{i \theta}\right)$ and $\beta=\omega\left(e^{i \theta}\right) . G(\beta)=F(\alpha)$, and $G^{\prime}(\beta) \neq 0$, so in some small neighborhood $N, \alpha \in N \subseteq \mathcal{Q}$, we can define the single-valued analytic function $k=G^{-1}(F)$ with $k(\alpha)=\beta$. Consider the path $r \rightarrow \rho\left(r e^{i \theta}\right), r \in[0,1)$, on $W$. It starts at $\tilde{a} \in \tilde{\Omega}$ when $r=0$ and $\mathfrak{h}$ is analytic along it; that is, the function element $(h, \Omega)$ has an analytic continuation along the projected path $r \rightarrow$ $(\pi \circ \rho)\left(e^{i \theta}\right) . k$ is clearly a further analytic continuation to a neighborhood of the endpoint $\alpha=(\pi \circ \rho)\left(e^{i \theta}\right)$. From the definition of $W$ we therefore see that $\lim _{r \rightarrow 1} \rho\left(r e^{i \theta}\right)$ is an interior point of $W$ lying over $\alpha$. But this contradicts the fact that $\rho$ is a covering map. The contradiction shows $E_{3}=\varnothing$. 
Finally, we use the fact that $G\left(e^{i \theta}\right) \notin F(U)$, a.e. Consider $e^{i \theta} \in E_{4}$ with $\alpha=$ $\phi\left(e^{i \theta}\right)$ and $\beta=\omega\left(e^{i \theta}\right),|\beta|=1$. Let $\Gamma$ be the path $r \rightarrow \omega\left(r e^{i \theta}\right)$ which ends at $\beta$. Because $G \circ \omega=F \circ \phi$, we see that $G$ has asymptotic value $F(\alpha) \in F(\mathcal{Q})$ along $\Gamma$. Thus, at $\beta \in \partial \mathcal{U}$, precisely one of the following holds: $G$ has no nontangential limit, $G$ has a nontangential limit in $F(\mathscr{Q})$, or $G$ has a nontangential limit different from the asymptotic value along $\Gamma$. Each of these can occur only on a set of measure zero. That is, $\omega\left(E_{4}\right)$ lies in a subset of $\partial \mathscr{Q}$ of measure zero. An application of Lemma 2 shows $m\left(E_{4}\right)=0$ and completes the proof.

This proof gives one direction of the following result; the converse is straightforward.

Proposition. $F \stackrel{w}{\prec} G$ if and only if some choice of $G^{-1}(F)$, not the identity function, can be continued analytically to the Riemann surface associated with an inner function.

Of course, we are fortunate that a simple condition like $F<G$ actually allows us to determine the behavior of $G^{-1}(F)$. It gives hope that there are other situations in which one can recognize that weak subordination occurs; for example, there may be local geometric criteria analogous to the global condition $F<G$.

We record here a curious result which follows from Theorem 1 and the fact that the composition of two inner functions is itself an inner function (which follows, incidently, from Lemmas 2 and 4).

Corollary. Assume $F$ and $G$ are inner functions. Then there exist inner functions $\phi$ and $\omega$ such that $F \circ \phi=G \circ \omega$.

\section{Alternate approach.}

THEOREM 2. If $F<G$ then there is a plane region $R$ and a universal covering map $H:$ Q $\rightarrow R$ so that $F \stackrel{w}{\prec} H$ and $H \stackrel{w}{\prec} G$.

Proof. We must exhibit inner functions $\phi$ and $\psi$ and analytic functions $\nu$ and $\omega$ with $F \circ \phi=H \circ \nu$ and $H \circ \psi=G \circ \omega$. The basic idea is to modify $F$ and $G$ slightly (by composition with $\phi$ and $\omega$, respectively) so that the modified functions have a common range, $R$. Then we observe that each is subordinate (by $\nu$ and $\psi$, respectively) to the universal covering map $(H)$ of their common range. We proceed by constructing each of the required functions.

Construction of $\omega$. Let $E=S^{2} \backslash F(थ)$. $E$ is closed, $S^{2} \backslash E$ is connected, and by hypothesis, $G(\mathcal{Q}) \cap\left(S^{2} \backslash E\right)$ is nonempty. First, observe that $E$ has positive capacity since $G\left(e^{i \theta}\right) \in E$, a.e. [13, p. 210]. (Note the importance here of the assumption that $G$ has nontangential versus only radial limits. There are examples of nonconstant meromorphic functions on $\mathcal{Q}$ having limit 0 on almost every radius. See [5, §8.1].) Let $\Omega \subseteq \mathcal{Q}$ be a (connected) component of $G^{-1}\left(S^{2} \backslash E\right)$. If $\omega$ : $\mathcal{U} \rightarrow \Omega$ is a universal covering map, then $G \circ \omega$ : $\mathcal{U} \rightarrow S^{2} \backslash E$. Since $E$ has positive capacity, $G \circ \omega \in \mathrm{BC}$ [10, Chapter VII, §5], hence $G \circ \omega$ has nontangential limits, a.e. Since $G(\xi) \in E$ for all $\xi \in \partial \Omega \cap \mathscr{Q}$ and for almost all $\xi \in \partial \Omega \cap \partial \mathscr{Q}$, Lemma 4 implies $(G \circ \omega)\left(e^{i \theta}\right) \in E$, a.e. In particular, $F<(G \circ \omega)$. 
Construction of $\phi$. We begin with some notation and an extension of Frostman's theorem due to Rudin.

If $g \in \mathrm{BC}$ and $\alpha \in \mathbf{C}$, then $g-\alpha$ may be factored as

$$
g-\alpha=I_{\alpha} \cdot H_{\alpha} / J_{\alpha}
$$

where $I_{\alpha}$ and $J_{\alpha}$ are inner functions with no common inner factor and $H_{\alpha}$ is outer, $\left|H_{\alpha}\left(e^{i \theta}\right)\right|=\left|g\left(e^{i \theta}\right)-\alpha\right|$, a.e. [6, Chapter 2]. We refer to $I_{\alpha}$ as the inner factor of $g-\alpha$.

LEMma 5 (Rudin [5]). If $g \in \mathrm{BC}$, then the set of points $\alpha \in \mathbf{C}$ for which the inner factor of $g-\alpha$ has a nontrivial singular inner factor is of capacity zero.

Proof. Rudin's proof applies to functions which are in $\mathcal{H}(थ)$. Suppose $g \in \mathrm{BC}$ is meromorphic with the set of poles $A$. If $\phi_{A}: \mathcal{U} \rightarrow \mathscr{U} \backslash A$ is a universal covering map, then $\phi_{A}$ is an inner function by Lemma 3 and $g \circ \phi_{A} \in \mathcal{H}(\mathcal{U})$. If $I$ is a nontrivial singular inner factor of $g-\alpha$, then $I \circ \phi_{A}$ is a nontrivial singular inner factor for $g \circ \phi_{A}-\alpha$; so our conclusion follows from the holomorphic case.

LEMMA 6. If $g \in \mathrm{BC}$ and $f<g$, then the set $f(थ) \backslash g(थ)$ has capacity zero.

Proof. Let $B=\partial(g(\mathscr{U})) \cap f(\mathscr{U})$. We show $B$ has capacity zero. Then $f(\mathscr{U})$, being open and connected, cannot be separated by $B$, so we must have

$$
B=f(थ) \backslash g(थ),
$$

and we are done.

Pick $\alpha \in B$. Then $g-\alpha=I_{\alpha} \cdot H_{\alpha} / J_{\alpha}$. Choose $\delta>0$ so small that $\{|z-\alpha|<$ $\delta\} \subseteq f(\mathcal{Q}) . \quad f<g \Rightarrow\left|g\left(e^{i \theta}\right)-\alpha\right|>\delta$, a.e. $\Rightarrow\left|H_{\alpha}\left(e^{i \theta}\right)\right|>\delta$, a.e. $\Rightarrow\left|H_{\alpha}(z)\right|>\delta$, $z \in \mathcal{Q} \Rightarrow$

$$
\left|\frac{H_{\alpha}}{J_{\alpha}}(z)\right|>\delta, \quad z \in \text { थ. }
$$

Consider $I_{\alpha}$ : It has no zeros since $\alpha \notin g(\mathscr{Q})$; but $|g-\alpha|$ is not bounded away from zero since $\alpha \in \partial(g(\mathscr{Q}))$. In view of (4) we can only conclude that $I_{\alpha}$ is a nontrivial singular inner function. An application of Lemma 5 gives us the result.

Now to construct $\phi$ : We have $F<(G \circ \omega)$ and $G \circ \omega \in \mathrm{BC}$. Let

$$
B=F(\mathscr{Q}) \backslash(G \circ \omega)(\mathcal{Q}) \text { and } A=F^{-1}(B) \text {. }
$$

By Lemma $6, B$ has capacity zero, so $A \subset \mathcal{Q}$ is a closed set of capacity zero. Define $\phi: \mathcal{U} \rightarrow \mathcal{Q} \backslash A$ to be a universal covering map. By Lemma 3, $\phi$ will be an inner function. Note that

$$
F(\mathscr{U}) \backslash G(\mathscr{U}) \subseteq F(\mathscr{U}) \backslash(G \circ \omega)(\mathcal{U}),
$$

so $F(\mathscr{U}) \backslash G(\mathscr{Q})$ has capacity zero.

Construction of $H, \psi$, AND $\nu$. Observe that $G \circ \omega$ and $F \circ \phi$ have precisely the same range $R \subset S^{2}$. Since $E \subseteq S^{2} \backslash R$ has positive capacity, $\mathcal{U}$ is the universal covering surface of $R$. Define $H: \mathcal{Q} \rightarrow R$ to be a universal covering map; then the usual subordination principle gives analytic functions $\psi, \nu: \mathcal{Q} \rightarrow \mathcal{Q}$ so that

$$
G \circ \omega=H \circ \psi \text { and } F \circ \phi=H \circ \nu \text {. }
$$


Note that $\psi$ is an inner function. If not, then $H \circ \psi$ would have radial limits in the interior of $R$ on a set with positive measure, contradicting the fact that $H \circ \psi=$ $G \circ \omega$ has almost all its radial limits in $E \subseteq S^{2} \backslash R$.

Corollary. Let $G \in \mathfrak{T}(\mathscr{Q})$ with range $R=G(\mathscr{U})$ and assume $G$ has nontangential limits $G\left(e^{i \theta}\right)$ which lie outside $R$, a.e. Then $G \in \mathrm{BC}$ and $G=H \circ \psi$ where $H: \mathscr{U} \rightarrow R$ is a universal covering map and $\psi: \mathcal{Q} \rightarrow \mathcal{Q}$ is an inner function.

We may now show that the relations denoted by $<$ and $\stackrel{w}{\prec}$ are transitive within the nonconstant meromorphic functions. (It is easily seen that this can fail if constant functions are included.)

Assume $F, G, K \in \mathfrak{T}(\mathcal{Q})$ are nonconstant with $F<G$ and $G<K$. As noted in the previous proof, $G(\mathscr{U}) \backslash K(\mathscr{U})$ has capacity zero. Since $F(\mathscr{U}) \cap G(\mathscr{U})$ is a nonempty open set, we see that $F(\mathscr{U}) \cap K(\mathscr{U})$ is nonempty. Let $E_{1}=\left\{e^{i \theta}\right.$ : $\left.K\left(e^{i \theta}\right) \in F(\mathcal{Q}) \cap G(\mathcal{Q})\right\}$ and $E_{2}=\left\{e^{i \theta}: K\left(e^{i \theta}\right) \in F(\mathcal{U}) \backslash G(\Omega)\right\}$. Because $G<K$, $m\left(E_{1}\right)=0$, and because $F(थ) \backslash G(U)$ has capacity zero, $m\left(E_{2}\right)=0$. Thus $K\left(e^{i \theta}\right)$ $\notin F(\mathcal{U})$, a.e. We conclude that $F<K$.

Regarding weak subordination, suppose $F, G, K \in \mathfrak{N}(\mathcal{Q})$ are nonconstant with $F \stackrel{w}{\prec} G$ and $G \stackrel{w}{\prec} K$. There are then inner functions $\phi_{1}, \phi_{2}$ and analytic functions $\omega_{1}, \omega_{2}: \mathscr{U} \rightarrow$ U so that

$$
F \circ \phi_{1}=G \circ \omega_{1}, \quad G \circ \phi_{2}=K \circ \omega_{2} .
$$

Since $\phi_{2}$ is an inner function, we have $\omega_{1}<\phi_{2}$; so Theorem 1 implies the existence of an inner function $\alpha$ and analytic function $\beta$ : $\mathcal{Q} \rightarrow \mathcal{Q}$ with $\omega_{1} \circ \alpha=\phi_{2} \circ \beta$. In particular, $F \circ \phi_{1} \circ \alpha=G \circ \omega_{1} \circ \alpha=G \circ \phi_{2} \circ \beta=K \circ \omega_{2} \circ \beta$. The composition of inner functions $\phi_{1} \circ \alpha$ is itself an inner function, so the relation $F \circ\left(\phi_{1} \circ \alpha\right)=$ $K \circ\left(\omega_{2} \circ \beta\right)$ proves that $F \stackrel{w}{\prec} K$.

4. BMOA is stable. In [17] it was shown that the Hardy spaces $H^{p}, 0<p \leqslant \infty$, the Smirnov class $N_{*}$, and $\mathrm{BC}$ are stable, although this terminology was not used. We now investigate BMOA, analytic functions of bounded mean oscillation.

For the sake of completeness, we begin with an elementary proof that $H^{2}$ is stable. $\mathbb{Q}$ will be used to denote the group of conformal automorphisms (Möbius transformations) of $\mathscr{U} ;\langle\cdot, \cdot\rangle$ denotes the usual inner product in $\boldsymbol{H}^{2}$.

Proposition. $\mathrm{H}^{2}$ is stable.

Proof. It is well known that $H^{2}$ is closed under subordination. Thus, we need only show that if $\phi$ is an inner function, $f \in \Re(Q)$, and $f \circ \phi \in H^{2}$, then $f \in H^{2}$. It is not difficult to show, composing with elements from $\mathcal{Q}$ if necessary, that we may reduce to the case that $\phi(0)=0$.

The powers of $\phi,\left\{1, \phi, \phi^{2}, \ldots\right\}$, form an orthonormal set in $H^{2}$; let $S$ be its closed linear span with orthonormal projection $P: H^{2} \rightarrow S$. Define

$$
g(z)=\sum_{n=0}^{\infty}\left\langle f \circ \phi, \phi^{n}\right\rangle z^{n} .
$$


Since $\Sigma\left|\left\langle f \circ \phi, \phi^{n}\right\rangle\right|^{2} \leqslant\|f \circ \phi\|_{2}^{2}, g$ is in $H^{2}$ and $g \circ \phi=P(f \circ \phi)$. We show that $g \equiv f$

Let $h=f-g$ and suppose $h \neq 0$. $h$ cannot have a pole at $z=0$, since $h \circ \phi \in$ $H^{2}$, so we may write $h(z)=z^{k} h_{1}(z)$, some $k \geqslant 0$ and $h_{1}(0) \neq 0$. This gives

$$
\left\langle h \circ \phi, \phi^{k}\right\rangle=\left\langle\phi^{k}\left(h_{1} \circ \phi\right), \phi^{k}\right\rangle=\left\langle h_{1} \circ \phi, 1\right\rangle=\left(h_{1} \circ \phi\right)(0)=h_{1}(0) \neq 0 .
$$

However, this is a contradiction since $(h \circ \phi)=(f \circ \phi)-(g \circ \phi)=(f \circ \phi)-$ $P(f \circ \phi)$ is orthogonal to $S$. We conclude that $h \equiv 0$.

Definition (see [12]). For $f \in H^{2}$ and $\gamma \in Q$, let $f_{\gamma}$ denote the function $f \circ \gamma-(f \circ \gamma)(0)$. BMOA is the space of functions $f$ in $H^{2}$ with the property that

$$
M(f)=\sup _{\gamma \in \mathbb{Q}}\left\{\left\|f_{\gamma}\right\|_{2}\right\}<\infty .
$$

THEOREM 3. BMOA is stable.

Proof. Suppose $f \in$ BMOA and $\omega: \mathcal{Q} \rightarrow \mathcal{Q} . f \in \mathrm{BMOA} \Rightarrow f \in H^{2} \Rightarrow f \circ \omega \in$ $H^{2}$. For $\gamma \in \mathbb{Q}$, choose $\psi \in \mathbb{Q}$ so that $\left(\psi^{-1} \circ \omega \circ \gamma\right)(0)=0$. Then

$$
\left\|(f \circ \omega)_{\gamma}\right\|_{2}=\left\|\left(f \circ \psi \circ \psi^{-1} \circ \omega\right)_{\gamma}\right\|_{2} \leqslant\left\|f_{\psi}\right\|_{2} \leqslant M(f)<\infty,
$$

where the first inequality follows from Lemma 1 . Since this holds for all $\gamma \in \mathbb{Q}$, we conclude that $f \circ \omega \in$ BMOA. In the other direction, suppose $\phi$ is an inner function, $f \in \mathcal{N}(\mathcal{Q})$, and $f \circ \phi \in$ BMOA. First, $f \circ \phi \in H^{2}$ implies $f \in H^{2}$ because $H^{2}$ is stable. For $\gamma \in \mathbb{Q}, \gamma^{-1} \circ \phi$ is inner and hence assumes some value $\alpha$ with $(1+|\alpha|) /(1-|\alpha|)<4$ and $|f(\gamma(\alpha))-f(\gamma(0))|<1$. Say $\left(\gamma^{-1} \circ \phi\right)(\beta)=\alpha$, then choose $\psi \in \mathbb{Q}$ with $\psi(0)=\beta$, so that $\left(\gamma^{-1} \circ \phi \circ \psi\right)(0)=\alpha$. Then we have

$$
\begin{aligned}
\left\|f_{\gamma}\right\|_{2} & =\|f \circ \gamma-f(\gamma(0))\|_{2} \leqslant\|f \circ \gamma-f(\gamma(\alpha))\|_{2}+|f(\gamma(\alpha))-f(\gamma(0))| \\
& \leqslant 1+\left\|f \circ \gamma-\left(f \circ \gamma \circ \gamma^{-1} \circ \phi \circ \psi\right)(0)\right\|_{2} .
\end{aligned}
$$

By Lemma 1 , since $\gamma^{-1} \circ \phi \circ \psi$ is an inner function, this is

$$
\begin{aligned}
& \leqslant 1+\left(\frac{1+|\alpha|}{1-|\alpha|}\right)^{1 / 2}\left\|\left(f \circ \gamma \circ \gamma^{-1} \circ \phi \circ \psi\right)-\left(f \circ \gamma \circ \gamma^{-1} \circ \phi \circ \psi\right)(0)\right\|_{2} \\
& \leqslant 1+2\left\|(f \circ \phi)_{\psi}\right\|_{2} \leqslant 1+2 M(f \circ \phi)<\infty .
\end{aligned}
$$

Since this holds for all $\gamma \in \mathbb{Q}, f \in$ BMOA. This completes the proof.

REMARK. The range of a meromorphic function remains unchanged when the function is composed with an inner function, except for the possible removal of a set of capacity zero. Thus, stability seems to be a natural property to investigate for classes of functions where geometry of the ranges is involved. For the space $\mathscr{B}$ of Bloch functions, sets of capacity zero can be very important, and we will see in $\$ 6$ that $\mathscr{B}$ is not stable. On the other hand, sets of capacity zero are in some sense negligible for functions in BMOA, which is stable. The connection between geometry of the range of a function and membership in BMOA is investigated in [8]. In contrast, there are no geometric conditions on the range which imply a function is in VMOA, analytic functions of vanishing mean oscillation. This is reflected in the fact that VMOA is not stable-the identity function is VMOA, while most inner functions are not. 
5. The classes $M^{\Phi}$. Burkholder defined the classes $M^{\Phi}$ in terms of the nontangential maximal function; however this restricts consideration to holomorphic functions only. Fortunately, his work implies an equivalent definition based on balayage which, with a minor adjustment, allows meromorphic functions to enter in a natural way. We use the same notation $M^{\Phi}$ for this (possibly) broader class, with the justification that the original classes are precisely the holomorphic functions in $M^{\Phi}$. Indeed, we will see shortly that when $\Phi(\lambda)=\lambda^{p}, 0<p<\infty$, then the associated classes $M^{\Phi}$ (denoted $M^{p}$ in [4]) contain only holomorphic functions, so our definition is equivalent to Burkholder's. These specific classes are of particular interest because they generalize the $H^{p}$ spaces.

Before defining $M^{\Phi}$ we need some notation. A good reference for the potential theory terminology and results used in this section is Helms [9].

Fix $f \in \Re(\mathscr{U})$ and $\lambda>0$. $\delta$ denotes the superharmonic functions in $\mathscr{Q}$. Define sets

and the function

$$
V_{\lambda}^{f}=\{|f|<\lambda\}, \quad W_{\lambda}^{f}=\{|f| \geqslant \lambda\}
$$

$$
R_{\lambda}^{f}(z)=\inf \left\{v(z): v \in \mathcal{S}, v \geqslant 0, v \geqslant 1 \text { on } W_{\lambda}^{f}\right\}, \quad z \in \mathcal{Q} .
$$

Each point of $W_{\lambda}^{f}$ is contained in a continuum belonging to $W_{\lambda}^{f}$, so each component of $V_{\lambda}^{f}$ is a Dirichlet region. Also, the boundary of $W_{\lambda}^{f}$ in $\mathcal{Q}$ is a level set for the meromorphic function $f$, so each $z_{0} \in \partial W_{\lambda}^{f} \cap \mathcal{Q}$ has a neighborhood $N=$ $\left\{\left|z-z_{0}\right|<\varepsilon\right\}$ such that $N \cap V_{\lambda}^{f}$ has a finite number of components. Using standard arguments in potential theory one can verify that $R_{\lambda}^{f}$ has the following properties:

(a) $R_{\lambda}^{f} \equiv 1$ on $W_{\lambda}^{f}$.

(b) $R_{\lambda}^{f}$ is a continuous real superharmonic function on $\mathcal{Q}$ bounded by 1 .

(c) In each component $D$ of $V_{\lambda}^{f}, R_{\lambda}^{f}$ is the (Perron-Wiener-Brelot) solution of the Dirichlet problem with boundary values 1 on $\partial D \cap \mathcal{Q}$ and 0 on $\partial D \cap \partial \mathcal{Q}$.

In short, $R_{\lambda}^{f}$ is the balayage of 1 relative to $W_{\lambda}^{f}$ on $\mathcal{Q}$.

Definition. Let $\Phi$ be a real function defined for all large $\lambda$. To avoid trivialities assume $\Phi(\lambda)>\delta>0$ for some $\delta$ and all large $\lambda . M^{\Phi}$ is the class of functions $f \in \Re(U)$ with the property that

$$
\lim _{\lambda \rightarrow \infty} \inf \Phi(\lambda) R_{\lambda}^{f}(\alpha)=0
$$

for some $\alpha \in \mathcal{U}$ not a pole of $f$. This is independent of $\alpha$, for suppose $\beta \in \mathcal{U}$ is also not a pole of $f$. For sufficiently large $\lambda_{0}, \alpha$ and $\beta$ will be in the same component of $V_{\lambda_{0}}^{f}$, and for $\lambda>\lambda_{0}, R_{\lambda}^{f}$ will be positive and harmonic in $V_{\lambda_{0}}^{f}$. By Harnack's inequality there are constants $c$ and $C$, independent of $\lambda>\lambda_{0}$, so that

$$
c R_{\lambda}^{f}(\alpha) \leqslant R_{\lambda}^{f}(\beta) \leqslant C R_{\lambda}^{f}(\alpha) .
$$

Therefore, (5) holds or fails simultaneously for $\alpha$ and $\beta$.

If $F \in \mathcal{H}(\mathcal{Q})$, we may take $\alpha=0$ in (5). Referring to the proofs of Lemmas 1 and 4 in [4], we see that we arrive at the same collection of functions on $\mathcal{H}(\mathcal{U})$ as Burkholder. To see what changes may occur, consider $F(z)=1 / z$. For $\lambda>0$, 


$$
R_{\lambda}^{f}(z)=\log |1 / z| / \log \lambda .
$$

Set $\alpha=1 / 2$ and consider (5). Clearly, $f \in M^{\Phi}$ if and only if

$$
\liminf _{\lambda \rightarrow \infty} \Phi(\lambda)(\log \lambda)^{-1}=0 \text {. }
$$

For instance, if $\Phi(\lambda)=\sqrt{\log \lambda}$, then $f \in M^{\Phi}$, while if $\Phi(\lambda)=\lambda^{p}, 0<p<\infty$, then $f \notin M^{\Phi}$. In fact, easy computations along this same line show that if $\Phi(\lambda)=\lambda^{p}, 0<p<\infty$, then $M^{\Phi}$ contains only holomorphic functions; that is, our definition of $M^{\Phi}$ gives precisely the same class as considered in [4].

THEOREM 4. $M^{\Phi}$ is stable.

Proof. Suppose $\omega: \mathcal{Q} \rightarrow \mathscr{U}$ is analytic and $f \in \mathscr{N}(\mathcal{U})$. Then for $\alpha$ not a pole of $f \circ \omega$

$$
R_{\lambda}^{f \circ \omega}(\alpha) \leqslant R_{\lambda}^{f}(\omega(\alpha)) .
$$

Therefore, $f \in M^{\Phi} \Rightarrow f \circ \omega \in M^{\Phi}$. The other condition for stability is more difficult. It is sufficient to prove that when $\omega=\phi$, an inner function, then equality holds in (6).

Fix $\lambda>0$. If $|(f \circ \phi)(\alpha)| \geqslant \lambda$, then each side of (6) is 1 and we are done. Hence we assume $\lambda$ is sufficiently large so that $\phi(\alpha) \in V_{\lambda}^{f}$. Denote by $D$ the component of $V_{\lambda}^{f}$ containing $\phi(\alpha)$. For each $r,|\phi(\alpha)|<r<1$, denote by $D_{r}$ the component of $D \cap\{|z|<r\}$ containing $\phi(\alpha)$ and by $\Omega_{r}$ the component of $\phi^{-1}\left(D_{r}\right)$ containing $\alpha$. Both $D_{r}$ and $\Omega_{r}$ are Dirichlet regions. Let $u_{r}$ be the solution to the Dirichlet problem in $D_{r}$ with boundary values 1 on $\partial D_{r} \cap \partial V_{\lambda}^{f}$ and 0 elsewhere. Define $v_{r}$, harmonic on $\Omega_{r}$, by $v_{r}=u_{r} \circ \phi$.

First we consider the functions $v_{r}$. Suppose $z_{0} \in \partial \Omega_{r}$; then one and only one of the following occurs:

(a) $z_{0} \in \partial \mathscr{Q}$. Because $\phi$ is an inner function, $\partial \Omega_{r} \cap \partial \mathscr{Q}$ has harmonic measure zero with respect to $\Omega_{r}$. (See the proof of Proposition 3.3 in [17].) Thus we can ignore this part of $\partial \Omega_{r}$.

(b) $z_{0} \in \mathcal{Q}$ and $\phi\left(z_{0}\right) \in \partial V_{\lambda}^{f}$. In this case, $z_{0} \in \partial V_{\lambda}^{f \circ \phi}$, and

$$
\limsup _{\substack{z \rightarrow z_{0} \\ z \in \Omega_{r}}} v_{r}(z) \leqslant 1=R_{\lambda}^{f \circ \phi}\left(z_{0}\right) .
$$

(c) $z_{0} \in \mathscr{Q}$ and $\phi\left(z_{0}\right) \notin \partial V_{\lambda}^{f}$. In this case, $\left|\phi\left(z_{0}\right)\right|=r$, so $u_{r}$ has continuous boundary values in a neighborhood of $\phi\left(z_{0}\right)$. Therefore,

$$
\lim _{\substack{z \rightarrow \phi\left(z_{0}\right) \\ z \in D_{r}}} u_{r}(z)=0
$$

which gives

$$
\limsup _{\substack{z \rightarrow z_{0} \\ z \in \Omega_{r}}} v_{r}(z)=0 \leqslant R_{\lambda}^{f \circ \phi}\left(z_{0}\right) .
$$

Now, (7) and (8) and the fact that $v_{r}$ and $R_{\lambda}^{f \circ \phi}$ are bounded imply $v_{r}<R_{\lambda}^{f \circ \phi}$ in $\Omega_{r}$. In particular, we have shown

$$
v_{r}(\alpha)=u_{r}(\phi(\alpha)) \leqslant R_{\lambda}^{f \circ \phi}(\alpha), \quad|\phi(\alpha)|<r<1 .
$$


Next we consider the functions $u_{r}$. As $r \uparrow 1, D_{r}$ increases to $D$, and for $r_{1}<r_{2}$ it is clear that $u_{r_{1}} \leqslant u_{r_{2}} \leqslant R_{\lambda}^{f}$ on $D_{r_{1}}$. Applying Harnack's theorem, there exists a harmonic function $u \geqslant 0$ on $D$ with

$$
u_{r} \uparrow u
$$

Of course,

$$
u \leqslant R_{\lambda}^{f} \text { on } D \text {. }
$$

Fix $z_{0} \in \partial D \cap \mathscr{Q}$. As was observed earlier, there exists a neighborhood $N=$ $\left\{\left|z-z_{0}\right|<\varepsilon\right\}$ so that $D \cap N$ has a finite number of components. For sufficiently large $r$, all of those contained in $D$ will be contained in $D_{r}$ and $z_{0} \in \partial D_{r}$. The boundary values specified for $u_{r}$ will be continuous at $z_{0}$, so

$$
\lim _{\substack{z \rightarrow z_{0} \\ z \in D_{r}}} u_{r}(z)=1
$$

Since $D \cap N \subset D_{r}$,

$$
\liminf _{\substack{z \rightarrow z_{0} \\ z \in D}} u(z)=\lim _{\substack{z \rightarrow z_{0} \\ z \in D_{r}}} u(z) \geqslant \liminf _{\substack{z \rightarrow z_{0} \\ z \in D_{r}}} u_{r}(z)=1
$$

Obviously, if $z_{0} \in \partial D \cap \partial \mathscr{Q}$,

$$
\liminf _{\substack{z \rightarrow z_{0} \\ z \in D}} u(z) \geqslant 0 .
$$

But $R_{\lambda}^{f}$ is the solution of the Dirichlet problem in $D$ with boundary values 1 on $\partial D \cap \mathscr{Q}$ and 0 on $\partial D \cap \partial \mathscr{U}$. Therefore, (12) and (13) imply $R_{\lambda}^{f} \leqslant u$ on $D$. Along with (11) this gives

$$
u(\phi(\alpha))=R_{\lambda}^{f}(\phi(\alpha))
$$

Finally, (9), (10), and (14) imply equality in (6) with $\omega=\phi$. This concludes the proof that $M^{\Phi}$ is stable.

\section{Remarks.}

6.1. We give two examples of spaces which are not stable. The first is due to Richard Timoney; the second was considered at the suggestion of L. A. Rubel.

EXAMPLE I. We denote by $\mathscr{B}$ the Bloch functions. There are several characterizations of this space (see, for instance, [2]), but for our purposes the best description is as follows: Let $f \in \mathcal{H}(\mathcal{Q})$ and let $d_{f}(z)$ denote the radius of the largest schlicht disc about $f(z)$ on the Riemann image surface of $f$. Then $f \in \mathscr{B}$ if and only if $\sup _{z \in \mathcal{U}} d_{f}(z)<\infty$. Let $g$ be any meromorphic function not in $\mathscr{B}$. Let $\mathbf{Z}^{2}=\{z \in$ C: $\operatorname{Im} z$ and $\operatorname{Re} z$ are integers $\}$ and let $A=g^{-1}\left(\mathbf{Z}^{2} \cup\{\infty\}\right) . A \subset \mathscr{U}$ is closed and of capacity zero, hence a universal covering map $\phi_{A}$ : $U \rightarrow \mathcal{U} \backslash A$ is an inner function. Now $f=g \circ \phi_{A}$ is holomorphic and omits $\mathbf{Z}^{2}$, so $\sup _{z \in \mathcal{Q}} d_{f}(z) \leqslant \sqrt{2} / 2$. Therefore, $g \circ \phi_{A}=f \in \Re$ although $g \notin \mathscr{B}$, so $\mathscr{B}$ is not stable.

EXAMPLE II. Let $\rho$ be a continuous, decreasing, real function on $0 \leqslant r \leqslant 1$ with $\rho(1)=0$ and $\rho(r)>0$ for $r<1$. Let $\mathcal{X}$ be the linear space of functions $f \in \mathcal{H}(\mathcal{U})$ such that $f(z) \rho(|z|) \rightarrow 0$ as $|z| \rightarrow 1$. These spaces were introduced by L. A. Rubel and A. L. Shields [14]. We show that $\mathcal{X}$ may fail to be stable. 


$$
\begin{aligned}
& \text { Let } \rho(r)=\exp \{(r+1) /(r-1)\}, 0 \leqslant r \leqslant 1 \text {. Consider } \\
& f(z)=\exp \left\{-\frac{3}{2}((z+1) /(z-1))\right\},
\end{aligned}
$$

and let $\phi$ be the Möbius transformation $\phi(z)=\left(z-\frac{1}{2}\right) /\left(1-\frac{1}{2} z\right)$. Then $|f(r)| \rho(r)$ $\rightarrow \infty$ as $r \rightarrow 1$, so $f \notin \mathcal{X}$. However, $f \circ \phi=\exp \left\{-\frac{1}{2}((z+1) /(z-1))\right\} \cdot|(f \circ \phi)(z)|$ $\leqslant(f \circ \phi)(|z|), z \in \mathcal{U}$, and $|f(\phi(r))| \rho(r) \rightarrow 0$ as $r \rightarrow 1$, so $f \circ \phi \in \mathcal{X}$.

6.2. In [4], Burkholder considers classes, $M_{\Psi}^{\Phi}$, more general than those we consider in $\$ 5$. In fact, we can define analogous classes for any upper semicontinuous function $\Psi$ on $\mathbf{C}$; however, it is no longer clear that these are all stable.

To be specific, if $\Psi$ is upper semicontinuous we may define $M_{\Psi}^{\Phi}$ to be the class of $f \in \mathfrak{N}(\mathcal{Q})$ such that

$$
\liminf _{\lambda \rightarrow \infty} \Phi(\lambda) R_{\lambda}^{\Psi(f)}(\alpha)=0,
$$

for some $\alpha$ not a pole of $f$. The proof in $\$ 5$ shows that a simple sufficient condition for $M_{\Psi}^{\Phi}$ to be stable is that $\Psi$ have the following property:

Given $f \in \mathfrak{T}(\mathscr{U})$ and $\lambda$ sufficiently large, if $\left|\Psi\left(f\left(z_{0}\right)\right)\right|=\lambda, z_{0} \in \mathcal{U}$, then there is a neighborhood $N=\left\{\left|z-z_{0}\right|<\varepsilon\right\}$ such that

$$
N \cap\{|\Psi(f)|<\lambda\}
$$

has a finite number of components.

This holds, for example, if $\Psi(z)=\operatorname{Re} z, \Psi(z)=(\operatorname{Re} z)^{+}$, or $\Psi(z)=\log ^{+}|z|$. Is some such condition necessary, or are all such classes $M_{\Psi}^{\Phi}$ stable?

6.3. If was proven in [3] that if $F<G, F(0)=G(0)$, and $G \in H^{p}, 0<p<\infty$, then not only is $F \in H^{p}$, but $\|F\|_{p} \leqslant\|G\|_{p}$. This follows easily from Theorem 1: If $\alpha=G(0)=F(0)$, then in constructing the functions $\phi$ and $\omega$ we may specify $\phi(0)=0=\omega(0)$. The final statement in Lemma 1 holds for any value $0<p<\infty$ in place of 2 , hence

$$
\|F\|_{p}=\|F \circ \phi\|_{p}=\|G \circ \omega\|_{p} \leqslant\|G\|_{p} .
$$

The work in [17] shows that for $\mathcal{X}=H^{p}, 0<p \leqslant \infty, N_{*}$, or $\mathrm{BC}, \mathcal{X}$ actually has a property slightly stronger than stability. Namely, if $\phi$ is an inner function, $f$ is meromorphic on $\phi(\mathscr{Q})$, and $f \circ \phi \in \mathcal{X}$, then $f$ can be extended to be meromorphic on $\mathcal{Q}$ and $f \in \mathcal{X}$.

Since BMOA $\subseteq H^{2}$, this also holds for $\mathcal{X}=$ BMOA. However, it clearly does not hold for stable classes in general. For example, let $f$ be analytic in $\mathscr{U}$ except for an essential singularity at $z=0$, and let $\phi(z)=\exp \{(z+1) /(z-1)\}$. Then with a proper choice of the function $\Phi$ we can have $f \circ \phi \in M^{\Phi}$, even though $f \notin M^{\Phi}$. The stronger property only holds when sets of capacity zero are in some sense "removable" under the growth condition associated with the class $\mathcal{X}$.

\section{REFERENCES}

1. L. Ahlfors and L. Sario, Riemann surfaces, Princeton Univ. Press, Princeton, N. J., 1960.

2. J. M. Anderson, J. Clunie and Ch. Pommerenke, On Bloch functions and normal functions, J. Reine Angew. Math. 270 (1974), 12-37.

3. D. L. Burkholder, Exit times of Brownian motion, harmonic majorization, and Hardy spaces, Adv. in Math. 26 (1977), 182-205. 
4. (1978), 197-212.

Boundary value estimation of the range of an analytic function, Michigan Math. J. 25

5. E. F. Collingwood and A. H. Lohwater, The theory of cluster sets, Cambridge Tracts in Math. and Math. Phys., no. 56, Cambridge Univ. Press, New York, 1966.

6. P. L. Duren, Theory of $H^{P}$ spaces, Academic Press, New York, 1970.

7. L. J. Hansen, Boundary values and mapping properties of $H^{p}$ functions, Math. Z. 128 (1972), 189-194.

8. W. K. Hayman and Ch. Pommerenke, On analytic functions of bounded mean oscillation, Bull. London Math. Soc. 10 (1978), 219-224.

9. L. L. Helms, Introduction to potential theory, Interscience, New York, 1969.

10. R. Nevanlinna, Analytic functions, Die Grundlehren der mathematischen Wissenschaften, Band 162, Springer-Verlag, Berlin and New York, 1970.

11. E. Nordgren, Composition operators, Canad. J. Math. 20 (1968), 442-449.

12. Ch. Pommerenke, Schlichte Funktionen und analytische Funktionen von beschränkter mittlerer Oszillation, Comment. Math. Helv. 52 (1977), 591-602.

13. I. I. Priwalow, Randeigenschaften analytischer Funktionen, VEB Deutscher Verlag der Wissenschaften, Berlin, 1956.

14. L. A. Rubel and A. L. Shields, The second duals of certain spaces of analytic functions, J. Austral. Math. Soc. 11 (1970), 276-280.

15. W. Rudin, $A$ generalization of $a$ theorem of Frostman, Math. Scand. 21 (1967), 136-143.

16. $\ldots, L^{p}$-isometries and equimeasurability, Indiana Univ. Math. J. 25 (1976), 215-228.

17. K. Stephenson, Functions which follow inner functions, Illinois J. Math. 23 (1979), 259-266.

18. M. Tsuji, Potential theory in modern function theory, Chelsea, New York, 1975.

Department of Mathematics, University of Tennessee, Knoxville, Tennessee 37916 